\title{
Complex dynamical behaviors in a discrete eco-epidemiological model with disease in prey
}

\author{
Zengyun $\mathrm{Hu}^{1 *}$, Zhidong Teng ${ }^{2}$, Chaojun Jia ${ }^{1}$, Long Zhang ${ }^{2}$ and Xi Chen ${ }^{1 *}$
}

\author{
"Correspondence: \\ huzengyun@ms.xjb.ac.cn; \\ chenxi@ms.xjb.ac.cn \\ ${ }^{1}$ State Key Laboratory of Desert and \\ Oasis Ecology, Xinjiang Institute of \\ Ecology and Geography, Chinese \\ Academy of Sciences, Urumqi, \\ 830011, People's Republic of China \\ Full list of author information is \\ available at the end of the article
}

\begin{abstract}
In this study, the different dynamical behaviors caused by different parameters of a discrete-time eco-epidemiological model with disease in prey are discussed in ecological perspective. The results indicate that when we choose the same parameters and initial value and only vary the key parameters there appears a series of dynamical behaviors. For example, only varying the death rate of the infected prey (the carrying capacity of the environment for the prey population or the transmission coefficient), there appear chaos, Hopf (flip) bifurcation, local stability, flip (Hopf) bifurcation, and chaos; when only varying the predation coefficient there appear chaos, Hopf bifurcation, local stability, Hopf bifurcation, and chaos. These results are far richer than the corresponding continuous-time model and are rarely seen in previous works. Numerical simulations not only illustrate our results but also exhibit complex dynamical behaviors, such as period-doubling bifurcation in period-2,4,8, quasi-periodic orbits, 3,5,11,16-period orbits and chaotic sets. Moreover, the numerical simulations imply that when the death rate of the infected prey reaches a fixed value the disease dies out. Also, when the predation coefficient parameter reaches some value the disease dies out. These findings indicate that it is practicable to control the disease transmitting in prey by changing the death rate of the infected prey and the predation coefficient parameter.
\end{abstract}

Keywords: discrete eco-epidemiological model; predator-prey; flip bifurcation; Hopf bifurcation; chaos; complex dynamical behavior

\section{Introduction}

Generally, the ecological models are used to study the competitive, cooperation, and preypredator relationships between different species in nature [1-5]. And the epidemic models are used to detect the outbreak, transmission, and extinction characteristics of the different diseases [6-10]. Actually, the prey (or predator) species may infect diseases, and the diseases will spread among the prey and predator species. For example, in Salton Sea of California, the Tilapia fish is infected by a virio class of bacteria, Vibro alginolyticus, which spreads in the fish species and the infected fish become much easier available for predation for piscivorous birds $[11,12]$. Then the dynamical behaviors of the prey-predator relationship become more complex than before. For this case, we not only study the behavior characteristics in the prey-predator process but also consider the disease spreading in the

C2014 Hu et al.; licensee Springer. This is an Open Access article distributed under the terms of the Creative Commons Attribution License (http://creativecommons.org/licenses/by/2.0), which permits unrestricted use, distribution, and reproduction in any medium, provided the original work is properly cited. 
species. Therefore, the ecological theory should be combined with the epidemiological theory to explain the above ecological phenomena. That is the eco-epidemiology theory.

In the last few decades, as a new branch of theoretical biology the eco-epidemiology has received more and more attention (for example, [13-23] and the references cited therein). The reason is that the eco-epidemiology studied by different types of mathematical models can help us to understand the natural world well from ecology and epidemic perspectives [13]. Furthermore, we can control the transmission of diseases among different species through varying the key parameters when the dynamical behaviors of the corresponding models have been discussed clearly $[14,15]$.

Most of the above models are mainly based on the following cases: case 1, diseases transmit only in prey species (for example, [18-25] and the references cited therein); case 2, diseases transmit only in predator species (for example, see [26-30] and the references cited therein); case 3, diseases transmit both in prey and predator species (see [31]). The dynamical behaviors of the models with disease are studied, such as the stability, periodic solution, oscillation, bifurcation, and chaos. Their results indicated that the predators die out and the prey tends to its carrying capacity; or the infected prey and the predators both die out; or the predator and prey coexist.

The complex dynamical behaviors of discrete-time predator-prey models have already received much attention by lots of studies: such as stability, permanence, existence of periodic solutions, bifurcation, and chaos phenomenons [32-41]. We obtain a series of bifurcations of a discrete-time predator-prey model when we only vary the parameter $K$ which is more complex than the corresponding continuous-time model (see [33]). And the results are more reasonable in a biological perspective.

Until now, there are few papers to study the dynamical behaviors of discrete-time ecoepidemiological models. How many eco-epidemiological phenomena can be explained by discrete-time models which are not explained by continuous-time models? Is there more complex dynamical behavior in a discrete-time eco-epidemiological model than the continuous-time one as we obtained (see [33])? In this paper, motivated by the above works we will study a discrete-time predator-prey models with disease in prey which is obtained from the corresponding continuous-time model. Let us consider the following continuous-time predator-prey model with disease in prey described by differential equations studied by Xiao and Chen [24]:

$$
\begin{aligned}
& \frac{d S}{d t}=r S\left(1-\frac{S+I}{K}\right)-\beta S I, \\
& \frac{d I}{d t}=\beta S I-c I-\frac{b I Y}{m Y+I}, \\
& \frac{d Y}{d t}=-d Y+\frac{k b I Y}{m Y+I},
\end{aligned}
$$

where $S(t), I(t)$, and $Y(t)$ denote the population density of susceptible prey, infected prey and the population density of predator at time $t$, respectively. $r$ is the intrinsic birth rate of the prey population, $K$ is the carrying capacity of the environment for the prey population, $\beta$ is the transmission coefficient, $c$ is the death rate of the infected prey, $m$ is the ratiodependent rate, $b$ is the predation coefficient, $k$ is the coefficient in conversing prey into predator, and $d$ is the death rate constant of the predator. The parameters $r, K, \beta, c, b, m$, $d$ are positive constants and $0<k \leq 1$. The reasons why the predators only eat infected 
prey can be found in [24]. And the authors obtained the permanence, global stability, and Hopf bifurcation for model (1.1).

The following discrete-time model corresponding with model (1.1) is considered:

$$
\begin{aligned}
& S_{t+1}=S_{t} \exp \left[r\left(1-\frac{S_{t}+I_{t}}{K}\right)-\beta I_{t}\right], \\
& I_{t+1}=I_{t} \exp \left[\beta S_{t}-c-\frac{b Y_{t}}{m Y_{t}+I_{t}}\right], \\
& Y_{t+1}=Y_{t} \exp \left[\frac{k b I_{t}}{m Y_{t}+I_{t}}-d\right],
\end{aligned}
$$

where $r, K, \beta, c, b, m, d$, and $k$ are defined as in model (1.1). It is assumed that for the initial values of model (1.1) $S_{0}>0, I_{0}>0, Y_{0}>0$, and all the parameters are positive. Obviously, if the initial value $\left(S_{0}, I_{0}, Y_{0}\right)$ is positive, then the corresponding solution $\left(S_{t}, I_{t}, Y_{t}\right)$ is positive too.

In this paper, we will study the dynamical behaviors of model (1.2). The existence and local stability of equilibria, flip bifurcation, Hopf bifurcation, and chaos will be discussed by using the theory of difference equation. Moreover, by varying different parameters, the different dynamical behaviors will be studied for the same equilibrium and these phenomena also can be explained as regards their ecological significance. Finally, we will use the numerical simulations to indicate the correctness and rationality of our results.

The organization of this paper is as follows. In Section 2 we discuss the existence and local stability of equilibria in model (1.2). Furthermore, we study different bifurcations of model (1.2) caused by different parameters. In Section 3 we present the numerical simulations, which not only illustrate our results with the theoretical analysis, but also exhibit the complex dynamical behaviors such as the invariant cycle, 3,5,11,16-periodic solutions, flip bifurcation, Hopf bifurcation, and more than one attractors and chaotic sets. In the last section we give a discussion.

\section{Analysis of equilibria}

For model (1.2), we always assume that any solution $\left(S_{t}, I_{t}, Y_{t}\right)$ satisfies initial values $S_{0}>0$, $I_{0}>0$, and $Y_{0}>0$, and all the parameters $r, K, \beta, c, b, m, k$, and $d$ are positive. It is obvious that any solutions of model (1.2) are nonnegative for all $t \geq 0$.

Let $R_{0}=\frac{K \beta}{c}$, which is the basic reproductive rate of model (1.2). After some simple calculations, we first have the following results on the existence of the nonnegative equilibria of model (1.2).

\section{Theorem 1}

(1) When $R_{0} \leq 1$, model (1.2) has only an equilibrium $E_{1}(K, 0,0)$.

(2) When $R_{0}>1$, model (1.2) always has two equilibria $E_{1}(K, 0,0)$ and $E_{2}\left(\frac{c}{\beta}, \frac{r K}{r+K \beta}\left(1-\frac{c}{K \beta}\right), 0\right)$. Furthermore, if $k b>d$ and $m k(K \beta-c)-(k b-d)>0$, besides the two equilibria $E_{1}(K, 0,0), E_{2}\left(\frac{c}{\beta}, \frac{r K}{r+K \beta}\left(1-\frac{c}{K \beta}\right), 0\right)$, model (1.2) has a positive equilibrium $E^{*}\left(S^{*}, I^{*}, Y^{*}\right)$, where

$$
S^{*}=\frac{c m k+k b-d}{m k \beta}, \quad I^{*}=\frac{r}{r+K \beta}\left(K-S^{*}\right), \quad Y^{*}=\frac{k b-d}{m d} I^{*} .
$$


In order to obtain the stability of equilibria of model (1.2), we introduce the following lemmas.

Lemma 1 [36] Let $F(w)=w^{2}+B w+C$, where $B$ and $C$ are constants. Suppose $F(1)>0$ and $w_{1}, w_{2}$ are two roots of $F(w)=0$. Then

(1) $\left|w_{1}\right|<1$ and $\left|w_{2}\right|<1$ if and only if $F(-1)>0$ and $C<1$;

(2) $w_{1}=-1$ and $\left|w_{2}\right| \neq 1$ if and only if $F(-1)=0, B \neq 0,2$;

(3) $\left|w_{1}\right|<1$ and $\left|w_{2}\right|>1$ if and only if $F(-1)<0$;

(4) $\left|w_{1}\right|>1$ and $\left|w_{2}\right|>1$ if and only if $F(-1)>0$ and $C>1$;

(5) $w_{1}$ and $w_{2}$ are the conjugate complex roots and $\left|w_{1}\right|=\left|w_{2}\right|=1$ if and only if $B^{2}-4 C<0$ and $C=1$.

Lemma 2 [42] Let the equation $x^{3}+b x^{2}+c x+d=0$, where $b, c, d \in R$. Let further $A=$ $b^{2}-3 c, B=b c-9 d, C=c^{2}-3 b d$, and $\Delta=B^{2}-4 A C$. Then:

(1) The equation has three different real roots if and only if $\Delta \leq 0$.

(2) The equation has one real root and a pair of conjugate complex roots if and only if $\Delta>0$. Further, the conjugate complex roots are

$$
w=\frac{-2 b+Y_{1}^{\frac{1}{3}}+Y_{2}^{\frac{1}{3}}}{6} \pm i \frac{\sqrt{3}\left(Y_{1}^{\frac{1}{3}}-Y_{2}^{\frac{1}{3}}\right)}{6}
$$

where

$$
Y_{1,2}=b A+\frac{-B \pm \sqrt{B^{2}-4 A C}}{2} .
$$

Now, we study the stability of equilibria $E_{1}, E_{2}$, and $E^{*}$ of model (1.2). We first consider equilibrium $E_{1}(K, 0,0)$. The Jacobian matrix of model $(1.2)$ is

$$
J\left(E_{1}\right)=\left(\begin{array}{ccc}
1-r & -K\left(\beta+\frac{r}{K}\right) & 0 \\
0 & e^{K \beta-c} & 0 \\
0 & 0 & e^{-d}
\end{array}\right) .
$$

The three eigenvalues of $J\left(E_{1}\right)$ are

$$
w_{1}=1-r, \quad w_{2}=e^{K \beta-c}, \quad w_{3}=e^{-d} .
$$

When $R_{0}<1$ we have $0<w_{2}<1$ and when $R_{0}>1$ we have $\omega_{2}>1$. Hence, the local stability of $E_{1}(K, 0,0)$ is determined by $w_{1}$. Thus, we have the following result.

\section{Theorem 2}

(1) When $R_{0}<1$, we have the following conclusions.

(a) If $0<r<2$, then $E_{1}(K, 0,0)$ is a sink and locally asymptotically stable.

(b) If $r=2$, then $E_{1}(K, 0,0)$ is non-hyperbolic.

(2) When $R_{0}<1$ and $r>2$ or $R_{0}>1, E_{1}(K, 0,0)$ is unstable.

Furthermore, when $R_{0}<1,0<r<2$, the global asymptotically stable of $E_{1}(K, 0,0)$ is also can be obtained. In fact, from the first equation of model (1.2) and the positivity of the 
solution, we obtain

$$
S_{t+1}=S_{t} \exp \left[r\left(1-\frac{S_{t}+I_{t}}{K}\right)-\beta I_{t}\right]<S_{t} \exp \left[r\left(1-\frac{S_{t}}{K}\right)\right] .
$$

Let

$$
U_{t}=U_{t} \exp \left[r\left(1-\frac{U_{t}}{K}\right)\right]=U_{t} \exp \left[r-r \frac{U_{t}}{K}\right]
$$

Since $r<2$, by the conclusion (i) of Lemma 4 in [34], we obtain

$$
\lim _{t \rightarrow \infty} U_{t}=K
$$

Let $U(u)=u \exp \left[r-r \frac{u}{K}\right]$, then according to simple computing, $U$ is nondecreasing for $u \in\left(0, \frac{K}{r}\right]$. Two cases are considered for the global asymptotically stable of $E_{1}(K, 0,0)$.

Case 1: If $0<r \leq 1$, the conclusion (ii) of Lemma 4 in [34] shows that $\lim _{t \rightarrow \infty} U_{t} \leq \frac{K}{r}$, and from Lemma 7 in [34], we have $S_{t} \leq U_{t}$ for all $t \geq 2$, where $U_{t}$ is the solution of (2.2) with $S_{2}=U_{2}$. Consequently,

$$
\limsup S_{t} \leq \lim _{t \rightarrow \infty} U_{t}=K
$$

Then, for any constant $\epsilon>0$ sufficiently small there exists an integer $T>2$ such that if $t \geq T$, then $S_{t} \leq K+\epsilon$.

For $t>T$ and the second equation of model (1.2), we have

$$
I_{t+1}=I_{t} \exp \left[\beta S_{t}-c-\frac{b Y_{t}}{m Y_{t}+I_{t}}\right]<I_{t} \exp [\beta(K+\epsilon)-c]
$$

Since $R_{0}=\frac{\beta K}{c}<1$, the above $\epsilon$ can be chosen satisfied $\frac{\beta(K+\epsilon)}{c}<1$ and $\beta(K+\epsilon)-c<0$. Then $\lim _{t \rightarrow \infty} I_{t}=0$. From the third equation of model (1.2) $\lim _{t \rightarrow \infty} Y_{t}=0$. Hence, $\lim _{t \rightarrow \infty} S_{t}=K$. Equilibrium $E_{1}(K, 0,0)$ is global asymptotically stable.

Case 2: If $1<r<2$, by some simple computing we can easily obtain

$$
S_{t+1}<S_{t} \exp \left[r\left(1-\frac{S_{t}}{K}\right)\right] \leq \frac{K}{r} \exp [r-1]
$$

It means that $S_{t} \leq \frac{K}{r} \exp [r-1]$. For the second equation of model (1.2), if $\beta \frac{K}{r} \exp [r-1]-c<$ 0 , then $R_{0} \frac{\exp [r-1]}{r}<1$. We can easily obtain $\lim _{t \rightarrow \infty} I_{t}=0$. Consequently, by the other two equations of model (1.2) we have $\lim _{t \rightarrow \infty} Y_{t}=0$ and $\lim _{t \rightarrow \infty} S_{t}=K$. The global asymptotically stability of equilibrium $E_{1}(K, 0,0)$ is also obtained.

From the above discussion, we have the following result.

Theorem 3 If one of the following conditions hold, equilibrium $E_{1}(K, 0,0)$ is global asymptotically stable.

(1) $R_{0}<1$ and $0<r \leq 1$;

(2) $R_{0} \frac{\exp [r-1]}{r}<1$ and $1<r<2$. 
Next, we consider equilibrium $E_{2}\left(\frac{c}{\beta}, \frac{r K}{r+K \beta}\left(1-\frac{c}{K \beta}\right), 0\right)$. The Jacobian matrix of model (1.2) is

$$
J\left(E_{2}\right)=\left(\begin{array}{ccc}
1-\frac{r S}{K} & -S\left(\beta+\frac{r}{K}\right) & 0 \\
\beta I & 1 & -b \\
0 & 0 & e^{b k-d}
\end{array}\right)
$$

The corresponding characteristic equation of $J\left(E_{2}\right)$ is

$$
f(w)=\left(w-e^{k b-d}\right)\left(w^{2}+p w+q\right)
$$

where

$$
p=-\left(2-\frac{r S}{K}\right), \quad q=1-\frac{r S}{K}+\beta S I\left(\beta+\frac{r}{K}\right) .
$$

Obviously, $f(w)$ has one eigenvalue $w_{1}=e^{k b-d}$ and

(1) if $b k<d$, then $0<w_{1}<1$;

(2) if $b k=d$, then $w_{1}=1$;

(3) if $b k>d$, then $w_{1}>1$.

Let $g(w)=w^{2}+p w+q$. We denote by $\omega_{2,3}$ the two roots of equation $g(\omega)=0$. By simple computing, we obtain $g(1)>0$. Further,

$$
g(-1)=4-\frac{2 c r}{K \beta}+\frac{c r(K \beta-c)}{K \beta} .
$$

From $g(-1)=0$, we have

$$
K \beta=\frac{c r(c+2)}{4+c r}
$$

which equals

$$
r c^{2}+(2 r-K \beta r) c-4 K \beta=0
$$

Solving this equation we have

$$
\begin{aligned}
& c \triangleq c_{1}=\frac{K \beta r-2 r-\sqrt{(K \beta r-2 r)^{2}+16 K \beta r}}{2 r}, \\
& c \triangleq c_{2}=\frac{K \beta r-2 r+\sqrt{(K \beta r-2 r)^{2}+16 K \beta r}}{2 r} .
\end{aligned}
$$

Obviously, $c_{1}<0$. When $K \beta>\frac{c r(c+2)}{4+c r}$ or $0<c<c_{2}$, we have $F(-1)>0$. When $q=1$, we have $K \beta=1+c$. Hence, when $K \beta<1+c$, we obtain $q<1$ and when $K \beta>1+c$, we obtain $q<1$. Moreover, when $p \neq 0$, 2, we obtain $K \beta \neq 2 c r$, $4 c r$. When $p^{2}-4 q<0$, we obtain $(r-4) c<4$. Hence, by Lemma 1, we have

(1) if $\frac{c r(c+2)}{4+c r}<K \beta<1+c$, then $\left|w_{2,3}\right|<1$;

(2) if $K \beta=\frac{c r(c+2)}{4+c r}$ and $K \beta \neq 2 c r, 4 c r$, then $w_{2}=-1$ and $\left|w_{3}\right| \neq 1$ (or $w_{3}=-1$ and $\left|w_{2}\right| \neq 1$ );

(3) if $K \beta<\frac{c r(c+2)}{4+c r}$, then $\left|w_{2}\right|<1$ and $\left|w_{3}\right|>1$ (or $\left|w_{2}\right|>1$ and $\left|w_{3}\right|<1$ ); 
(4) if $K \beta>\max \left\{\frac{c r(c+2)}{4+c r}, 1+c\right\}$, then $\left|w_{2,3}\right|>1$;

(5) if $K \beta=1+c$ and $(r-4) c<4$, then $w_{2,3}$ are the conjugate complex roots with $\left|w_{2}\right|=\left|w_{3}\right|=1$.

From the above discussion, we have the following result.

Theorem 4 Let $R_{0}>1$, then we have the following conclusions.

(1) $E_{2}\left(\frac{c}{\beta}, \frac{r K}{r+K \beta}\left(1-\frac{c}{K \beta}\right), 0\right)$ is a sink and locally asymptotically stable if

$$
b k<d, \quad(r-4) c<4, \quad \frac{c r(c+2)}{4+c r}<K \beta<1+c ;
$$

(2) $E_{2}\left(\frac{c}{\beta}, \frac{r K}{r+K \beta}\left(1-\frac{c}{K \beta}\right), 0\right)$ is non-hyperbolic if one of the following conditions holds:
(A) $b k=d$
(B) $K \beta=\frac{c r(2+c)}{4+c r}$ and $K \beta \neq 2 c r, 4 c r$;
(C) $K \beta=1+c$ and $(r-4) c<4$;

(3) $E_{2}\left(\frac{c}{\beta}, \frac{r K}{r+K \beta}\left(1-\frac{c}{K \beta}\right), 0\right)$ is unstable if one of the following conditions holds:

(A) $b k \neq d$ and $K \beta>\max \left\{\frac{c r(c+2)}{4+c r}, 1+c\right\}\left(\right.$ or $\left.K \beta<\frac{c r(2+c)}{4+c r}\right)$;

(B) $b k>d$ and $\frac{c r(c+2)}{4+c r}<K \beta<1+c, r c<4+4 c$.

From the above discussion we also obtain:

(1) For equilibrium $E_{1}(K, 0,0)$, if $(b, c, d, k, m, r, \beta, K) \in M$, where

$$
M=\{(b, c, d, k, m, r, \beta, K): r=2, K \beta \geq c, b, c, d, k, m, \beta, K>0\},
$$

then one of the three eigenvalues of matrix $J\left(E_{1}\right)$ is -1 and the others are neither -1 nor 1 . Therefore, there may be a flip bifurcation at equilibrium $E_{1}(K, 0,0)$, if $r$ varies in the small neighborhood of $r=2$ and $(b, c, d, k, m, 2, \beta, K) \in M$.

(2) For equilibrium $E_{2}\left(\frac{c}{\beta}, \frac{r K}{r+K \beta}\left(1-\frac{c}{K \beta}\right), 0\right)$, if $(b, c, d, k, m, r, \beta, K) \in N$, where

$$
\begin{aligned}
N= & \left\{(b, c, d, k, m, r, \beta, K): K \beta=\frac{c r(2+c)}{4+c r}, K \beta \neq 2 c r, 4 c r,\right. \\
& b, c, d, k, m, r, \beta, K>0\}
\end{aligned}
$$

then one of the three eigenvalues of matrix $J\left(E_{2}\right)$ is -1 and the others are neither -1 nor 1 . Therefore, there may be a flip bifurcation at equilibrium $E_{2}$, if $K, \beta, c$, and $r$ vary in the small neighborhood of $K \beta=\frac{c r(2+c)}{4+c r}$ and $(b, c, d, k, m, r, \beta, K) \in N$.

Further, if $(b, c, d, k, m, r, \beta, K) \in P$, where

$$
\begin{aligned}
P= & \{(b, c, d, k, m, r, \beta, K): K \beta=1+c,(r-4) c<4, \\
& b, c, d, k, m, r, \beta, K>0\}
\end{aligned}
$$

then there are two conjugate eigenvalues $w_{2}$ and $w_{3}$ of matrix $J\left(E_{2}\right)$ with $\left|w_{2}\right|=\left|w_{3}\right|=1$. Therefore, there may be a flip bifurcation at equilibrium $E_{2}$, if $K, \beta$, and $c$ vary in the small neighborhood of $K \beta=1+c$ and $(b, c, d, k, m, r, \beta, K) \in P$.

Remark 1 From the above discussion, we further see that when we choose the same parameters and the initial value of model (1.2); then $K \beta$ shows a continuous increase from 
some fixed value above 0 to $\frac{c r(2+c)}{4+c r}$ then to $1+c$, then a series of different dynamical behaviors of equilibrium $E_{2}\left(\frac{c}{\beta}, \frac{r K}{r+K \beta}\left(1-\frac{c}{K \beta}\right), 0\right)$ will appear: chaos $\rightarrow$ flip bifurcation $\rightarrow$ local stability $\rightarrow$ Hopf bifurcation $\rightarrow$ chaos (see Figures 6 and 7) which is the same result as in [19].

Remark 2 Furthermore, we see that when we choose the same parameters and the initial value of model (1.2), then $c$ shows a continuous increase from some fixed value above 0 to $K \beta-1$ then to $c_{2}$, then a series of different dynamical behaviors at equilibrium $E_{2}\left(\frac{c}{\beta}, \frac{r K}{r+K \beta}\left(1-\frac{c}{K \beta}\right), 0\right)$ will appear: chaos $\rightarrow$ Hopf bifurcation $\rightarrow$ local stability $\rightarrow$ flip bifurcation $\rightarrow$ chaos too (see Figure 4). This phenomenon is rarely seen in previous work.

The above dynamical behaviors of model (1.2) will be displayed by the numerical simulations in Section 3.

Now, we consider the endemic equilibrium $E^{*}\left(S^{*}, I^{*}, Y^{*}\right)$. The Jacobian matrix of model (1.2) at endemic equilibrium $E^{*}\left(S^{*}, I^{*}, Y^{*}\right)$ is

$$
J\left(E^{*}\right)=\left(\begin{array}{ccc}
1-\frac{r S^{*}}{K} & -S^{*}\left(\beta+\frac{r}{K}\right) & 0 \\
\beta I^{*} & 1+\frac{b I^{*} Y^{*}}{\left(m Y^{*}+*^{*}\right)^{2}} & -\frac{b I^{* 2}}{\left(m Y^{*}+I^{*}\right)^{2}} \\
0 & \frac{b k \Psi^{*}{ }^{2}}{\left(m Y^{*}+I^{*}\right)^{2}} & 1-\frac{b k m I^{*} Y^{*}}{\left(m Y^{*}+I^{*}\right)^{2}}
\end{array}\right) .
$$

Let $d=\frac{b k I^{*}}{m Y^{*}+I^{*}}$. By simple computing, $J\left(E^{*}\right)$ is written in the following form:

$$
J\left(E^{*}\right)=\left(\begin{array}{ccc}
a_{11} & a_{12} & 0 \\
a_{21} & a_{22} & a_{23} \\
0 & a_{32} & a_{33}
\end{array}\right),
$$

where

$$
\begin{aligned}
& a_{11}=1-\frac{r(c m k+b k-d)}{m k K \beta}, \quad a_{12}=-\frac{(r+K \beta)(c m k+b k-d)}{m k K \beta}, \\
& a_{21}=\frac{r[m k K \beta-(c m k+b k-d)]}{m k(r+K \beta)}, \quad a_{22}=1+\frac{d(b k-d)}{b m k^{2}}, \\
& a_{23}=-\frac{d^{2}}{b k^{2}}, \quad a_{32}=\frac{(b k-d)^{2}}{b m k}, \quad a_{33}=1-\frac{d(b k-d)}{b k} .
\end{aligned}
$$

The corresponding characteristic equation of $J\left(E_{2}\right)$ can be written as

$$
F(w)=w^{3}+b_{1} w^{2}+b_{2} w+b_{3}=0,
$$

where

$$
\begin{aligned}
& b_{1}=-\left(a_{11}+a_{22}+a_{33}\right), \\
& b_{2}=a_{11}\left(a_{22}+a_{33}\right)+a_{22} a_{33}-a_{23} a_{32}-a_{12} a_{21}, \\
& b_{3}=-a_{11}\left(a_{22} a_{33}-a_{23} a_{32}\right)+a_{12} a_{21} a_{33} .
\end{aligned}
$$

Let

$$
A=b_{1}^{2}-3 b_{2}, \quad B=b_{1} b_{2}-9 b_{3}, \quad C=b_{2}^{2}-3 b_{1} b_{3}
$$


and

$$
\Delta=B^{2}-4 A C
$$

Further, we see that the derivative of $F(w)$ is

$$
F^{\prime}(w)=3 w^{2}+2 b_{1} w+b_{2} .
$$

Obviously, the equation $F^{\prime}(w)=0$ has two roots:

$$
w_{1,2}^{*}=\frac{1}{3}\left(-b_{1} \pm \sqrt{b_{1}^{2}-3 b_{2}}\right) .
$$

Furthermore,

$$
\left|w_{1,2}^{*}\right|^{2}=\frac{1}{9}\left(2 b_{1}^{2} \pm 2 b_{1} \sqrt{b_{1}^{2}-3 b_{2}}-3 b_{2}\right) .
$$

When $\Delta \leq 0$, by Lemma 2 , we see that equation (2.4) has three real roots $w_{1}, w_{2}$, and $w_{3}$. From this, we can easily prove that two roots $w_{1,2}^{*}$ of the equation $F^{\prime}(w)=0$ also are real.

When $\Delta>0$, by Lemma 2 , we see that equation (2.4) has one real root $w_{1}$ and a pair of conjugate complex roots $w_{2,3}$ :

$$
w_{2,3}=\frac{-2 b_{1}+Y_{1}^{\frac{1}{3}}+Y_{2}^{\frac{1}{3}}}{6} \pm i \frac{\sqrt{3}\left(Y_{1}^{\frac{1}{3}}-Y_{2}^{\frac{1}{3}}\right)}{6}
$$

with

$$
Y_{1,2}=b_{1} A+\frac{-B \pm \sqrt{B^{2}-4 A C}}{2} .
$$

Further, we have

$$
\begin{aligned}
F(1)= & 1+b_{1}+b_{2}+b_{3} \\
= & 1+a_{11}\left(a_{22}+a_{33}-a_{22} a_{33}+a_{23} a_{32}-1\right) \\
& +a_{12} a_{21}\left(a_{33}-1\right)-\left(a_{22}+a_{33}-a_{22} a_{33}+a_{23} a_{32}\right) \\
= & \frac{d r(c m k+k b-d)(k b-d)[m k K \beta-(c m k+k b-d)]}{b m^{2} k^{3} K \beta}>0
\end{aligned}
$$

and

$$
\begin{aligned}
F(-1)= & -1+b_{1}-b_{2}+b_{3} \\
= & -1-a_{11}\left(a_{22}+a_{33}+a_{22} a_{33}-a_{23} a_{32}+1\right) \\
& +a_{12} a_{21}\left(a_{33}+1\right)-\left(a_{22}+a_{33}+a_{22} a_{33}-a_{23} a_{32}\right) \\
= & \frac{r c_{*} c_{* *}}{m k K \beta}-\frac{r c_{*}\left(a_{33}+1\right)\left(m k K \beta-c_{*}\right)}{m^{2} k^{2} K \beta}-2 c_{* *},
\end{aligned}
$$


where

$$
\begin{aligned}
c_{*} & =c m k+k b-d, \\
c_{* *} & =a_{22}+a_{33}+a_{22} a_{33}-a_{23} a_{32}+1 \\
& =4+\frac{2 d(k b-d)(1-m k)}{b m k^{2}} .
\end{aligned}
$$

Since $F(1)>0$, on the dynamical properties of equilibrium $E^{*}\left(S^{*}, I^{*}, Y^{*}\right)$, we have the following result, which can be found in [43].

Theorem 5 Let $b k>d$ and $m k(K \beta-c)-(b k-d)>0$, then we have the following conclusions.

(1) If one of the following conditions hold, then $E^{*}\left(S^{*}, I^{*}, Y^{*}\right)$ is a sink and locally asymptotically stable.

(A) $\Delta \leq 0, F(-1)<0$, and $-1<w_{1,2}^{*}<1$.

(B) $\Delta>0, F(-1)<0$, and $\left|w_{2,3}\right|<1$.

(2) $E^{*}\left(S^{*}, I^{*}, Y^{*}\right)$ is non-hyperbolic if $F(-1)=0$ or $\Delta>0,\left|w_{2,3}\right|=1$.

(3) If the above conditions (1) and (2) do not hold, then $E^{*}\left(S^{*}, I^{*}, Y^{*}\right)$ is unstable.

The above discussion indicates that it is hard to obtain the values of parameters $b, k, r, \beta$, and $K$ when there exists a bifurcation at endemic equilibrium $E^{*}\left(S^{*}, I^{*}, Y^{*}\right)$ for model (1.2). From the ecological perspective, the predation coefficient parameter $b$ and the coefficient in conversing prey into predator $k$ are important for determining the dynamical behaviors when there exists an endemic equilibrium $E^{*}\left(S^{*}, I^{*}, Y^{*}\right)$ of model (1.2). Therefore, we will use the Matlab software to give the simulations as regards the complex dynamical behaviors of model (1.2) caused by the changing of $b$ and $k$ in Section 3.

\section{Numerical simulations}

In this section, we will give the bifurcation diagrams of model (1.2) to confirm the above theoretical analysis and show the new interesting complex dynamical behaviors by numerical simulation. Moreover, different dynamical behaviors caused by different parameters are discussed in the ecological perspective. For equilibrium $E_{1}(K, 0,0)$, we choose parameter $r$. For equilibrium $E_{2}\left(\frac{c}{\beta}, \frac{r K}{r+K \beta}\left(1-\frac{c}{K \beta}\right), 0\right)$, we choose four key parameters $r, c, K$, and $\beta$. Finally, the key parameters $b$ and $k$ are selected for positive equilibrium $E^{*}\left(S^{*}, I^{*}, Y^{*}\right)$.

Example 1 For detecting the dynamical behaviors of model (1.2) impacted by parameter $r$ (the intrinsic birth rate of $S_{t}$ ), we choose $b=0.2, c=0.6, d=0.12, k=0.1, m=0.2$, $\beta=0.05, K=8$, and $r \in[0.01,4]$ and initial value $\left(S_{0}, I_{0}, Y_{0}\right)=(4,0.5,0.1)$. It is obvious that $(b, c, d, k, m, \beta, K, r)=(0.2,0.6,0.12,0.1,0.2,0.05,8,2) \in M$. Then equilibrium $E_{1}(K, 0,0)=$ $E_{1}(8,0,0)$ and the flip bifurcation appears (Figure 1$)$.

Figure 1(A) suggests that when $0<r<2$ equilibrium $E_{1}(K, 0,0)$ is local stable and when $r=2, E_{1}(K, 0,0)$ loses its stability. When $r>2$ there exists a flip bifurcation. Moreover, a chaotic set is emerged with the increasing of $r$. However, the infected prey and the predator are always in extinction for any value of $r$, which can be seen from Figure 1(B) and (C). This result is agreement of the ecological perspective that when the infected prey is in extinction the predator certainly becomes in extinction. 


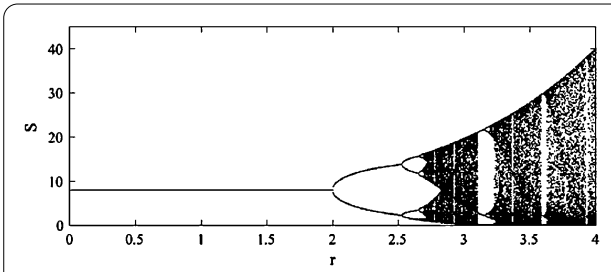

(A) Flip bifurcation of $S_{t}$ with $r \in[0.01,4]$

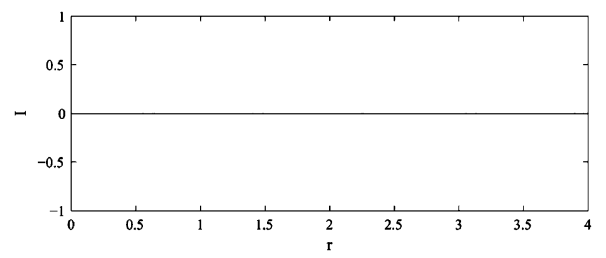

(B) Dynamical behavior of $I_{t}$ with $r \in[0.01,4]$

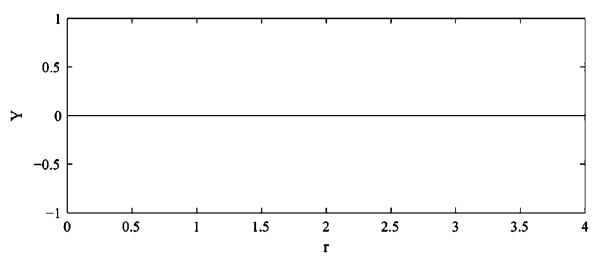

(C) Dynamical behavior of $Y_{t}$ with $r \in[0.01,4]$

Figure 1 The dynamical behaviors of $S_{t}-r, I_{t}-r$, and $Y_{t}-r$ with $b=0.2, c=0.6, d=0.12, k=0.1$, $m=0.2, \beta=0.05, K=8$, and $r \in[0.01,4]$, and the initial value $\left(S_{0}, I_{0}, Y_{0}\right)=(4,0.5,0.1)$ for model $(1.2)$.

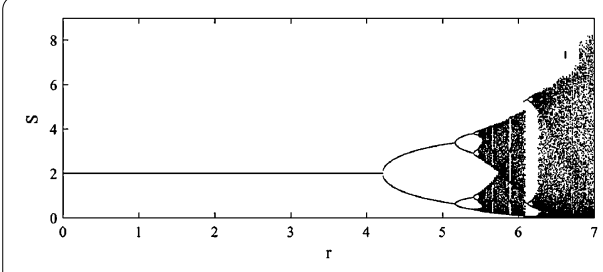

(A) Flip bifurcation of $S_{t}$ with $r \in[0.001,7]$

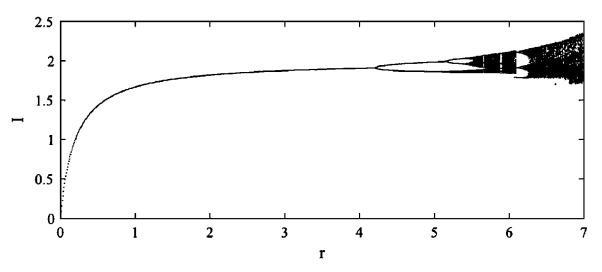

(B) Flip bifurcation of $I_{t}$ with $r \in[0.001,7]$

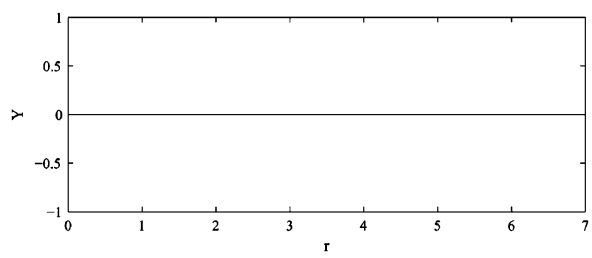

(C) Dynamical behavior of $Y_{t}$ with $r \in[0.001,7]$

Figure 2 The dynamical behaviors of $S_{t}-r, I_{t}-r$, and $Y_{t}-r$ with $b=0.15, c=0.1, d=0.2, k=0.2$, $m=0.3, \beta=0.05, K=4$, and $r \in[0.001,7]$, and the initial value $\left(S_{0}, I_{0}, Y_{0}\right)=(2,1,0.5)$ for model $(1.2)$.

Example 2 For detecting the dynamical behaviors of model (1.2) with equilibrium $E_{2}\left(\frac{c}{\beta}, \frac{r K}{r+K \beta}\left(1-\frac{c}{K \beta}\right), 0\right)$ impacted by parameter $r$, we choose $b=0.15, c=0.1, d=0.2, k=0.2$, $m=0.3, \beta=0.05, K=4$, and $r \in[0.001,7]$, and the initial value $\left(S_{0}, I_{0}, Y_{0}\right)=(2,1,0.5)$. It is obvious that $(b, c, d, k, m, \beta, K, r)=\left(0.15,0.1,0.2,0.2,0.3,0.05,4, \frac{80}{19}\right) \in N$. Then we have equilibrium $E_{2}\left(\frac{c}{\beta}, \frac{r K}{r+K \beta}\left(1-\frac{c}{K \beta}\right), 0\right)=E_{2}\left(2, \frac{2 r}{r+0.2}, 0\right)$, and there exists a flip bifurcation (Figure 2).

Figure 2 shows that equilibrium $E_{2}\left(2, \frac{2 r}{r+0.2}, 0\right)$ is stable when $0.001 \leq r<\frac{80}{19}$ and loses stability when $r=\frac{80}{19}$. Further, when $r>\frac{80}{19}$ there appears a flip bifurcation and chaos at equilibrium $E_{2}\left(2, \frac{2 r}{r+0.2}, 0\right)$. And a 3-periodic solution of model (1.2) appears when $r \approx 6.05$. However, $Y_{t}$ is in extinction for any value $r$ ultimately (Figure $2(\mathrm{C})$ ). 


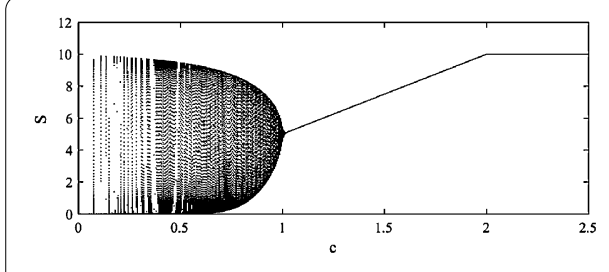

(A) Hopf bifurcation of $S_{t}$ with $c \in[0.01,2.5]$

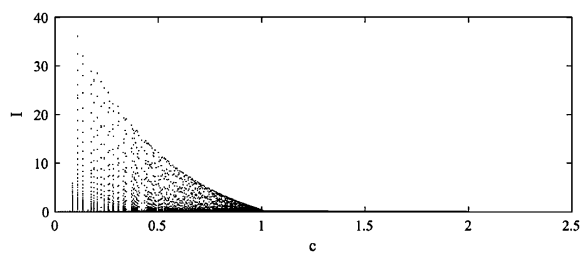

(C) Hopf bifurcation of $I_{t}$ with $c \in[0.01,2.5]$

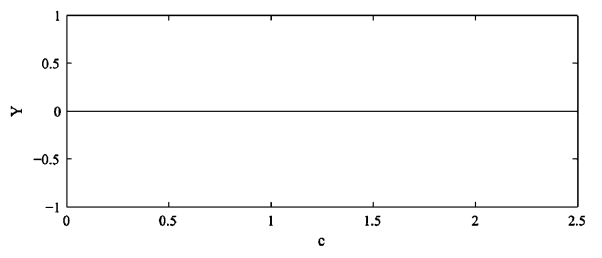

(B) Dynamical behaviors of $Y_{t}$ with $c \in[0.01,2.5]$

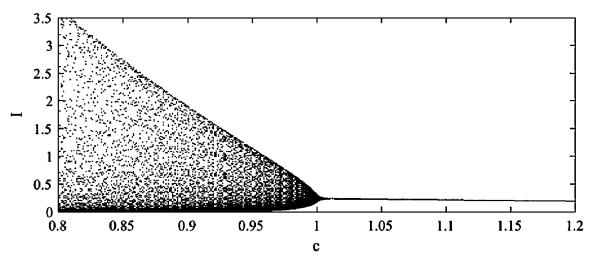

(D) The local amplification of $I_{t}$ with $c \in[0.8,1.2]$

Figure 3 The dynamical behaviors of $S_{t}-c, I_{t}-c$, and $Y_{t}-c$ with $b=0.1, d=0.6, k=0.1, m=0.5$, $r=0.1, \beta=0.2, K=10$, and $c \in[0.01,2.5]$ and the initial value $\left(S_{0}, I_{0}, Y_{0}\right)=(6,2,1)$ for model $(1.2)$.

Example 3 For detecting the dynamical behaviors of model (1.2) with equilibrium $E_{2}\left(\frac{c}{\beta}, \frac{r K}{r+K \beta}\left(1-\frac{c}{K \beta}\right), 0\right)$ impacted by parameter $c$ (the different death rate of infected prey), we choose two subcases of the parameters.

Subcase 1. Choosing $(b, d, k, m, r, \beta, K)=(0.1,0.6,0.1,0.5,0.1,0.2,10)$ and $c \in[0.01,2.5]$ and the initial value $\left(S_{0}, I_{0}, Y_{0}\right)=(6,2,1)$. It is obvious that $(b, d, k, m, r, \beta, K, c)=(0.1,0.6$, $0.1,0.5,0.1,0.2,10,1) \in P$. Then we have equilibrium $E_{2}\left(\frac{c}{\beta}, \frac{r K}{r+K \beta}\left(1-\frac{c}{K \beta}\right), 0\right)=E_{2}\left(5 c, \frac{5}{21}(2-\right.$ c), 0 ) and there exists a Hopf bifurcation (Figure 3).

From Figure 3, we find that there exists a Hopf bifurcation and chaos of model (1.2) when $0<c \leq 1$; When $1<c<2$ the number of $S_{t}$ is increasing and when $c \geq 2$ the number of $S_{t}$ is stable at 10. The number of $I_{t}$ is decreasing when $1<c<2$ and $I_{t}$ is becoming in extinction ultimately when $c \geq 2$. The number of $Y_{t}$ is always in extinction for any value of $c$ ultimately.

Subcase 2. Choosing $b=0.2, d=0.2, k=0.2, m=0.5, r=3, \beta=0.2, K=10$, and $c \in$ $[0.5,2.5]$, and the initial value $\left(S_{0}, I_{0}, Y_{0}\right)=(6,2,1)$. It is obvious that $(b, d, k, m, r, \beta, K, c)=$ $(0.2,0.2,0.2,0.5,3,0.2,10,1),(0.2,0.2,0.2,0.5,3,0.2,10,1.633) \in P, N$, respectively. Then we have equilibrium $E_{2}\left(\frac{c}{\beta}, \frac{r K}{r+K \beta}\left(1-\frac{c}{K \beta}\right), 0\right)=E_{2}(5 c, 6-3 c, 0)$ and there exist two bifurcation values of $c$ from the result of Theorem 3 . After some computing, we find that when $c=1$ and $c=1.633$ the Hopf bifurcation and flip bifurcation at equilibrium $E_{2}(5 c, 6-3 c, 0)$ appears, respectively (Figure 4).

From Figure 4(A)-(C), we find that there exist a Hopf bifurcation and chaos of model (1.2) when $0<c \leq 1$. When $1<c<1.633$ the number of $S_{t}$ is increasing, while the number of $I_{t}$ is decreasing. When $c \geq 1.633$ there appear the flip bifurcation and chaos of model (1.2). $I_{t}$ becomes in extinction when $c \approx 1.97$ (Figure 4(C)). Moreover, a 11-, 16- and 5-periodic solution appears when $c \approx 0.779, c \approx 0.81$, and $c \approx 0.87$, respectively (Figure 4(B)). However, $Y_{t}$ becomes in extinction because of $b k<d$ (Figure 4(D)).

Example 4 For detecting the dynamical behaviors of model (1.2) with equilibrium $E_{2}\left(\frac{c}{\beta}, \frac{r K}{r+K \beta}\left(1-\frac{c}{K \beta}\right), 0\right)$ impacted by parameter $K$ (the carrying capacity), we choose $b=0.1$, $c=0.4, d=0.2, k=0.2, m=0.5, r=0.2, \beta=0.2$, and $K \in[2,8]$, and the initial values 


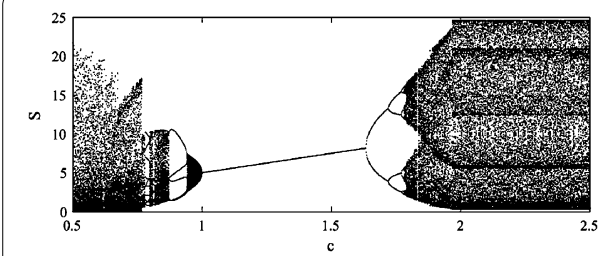

(A) Dynamical behaviors of $S_{t}$ with $c \in[0.5,2.5]$

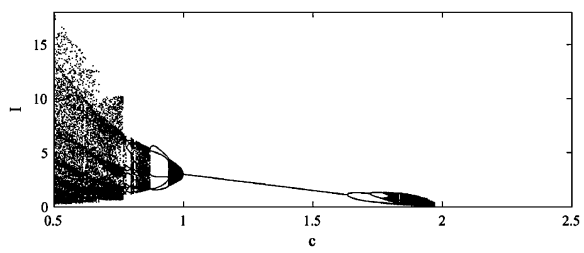

(C) Dynamical behaviors of $I_{t}$ with $c \in[0.5,2.5]$

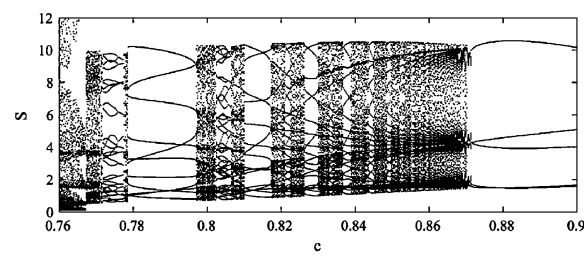

(B) The local amplification of $S_{t}$ with $c \in[0.76,0.9]$

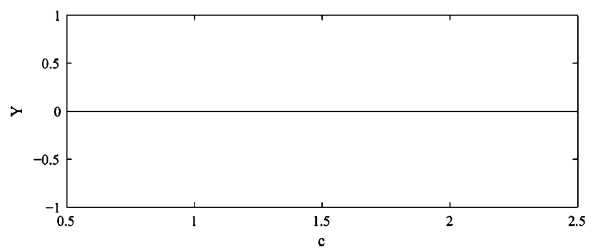

(D) Dynamical behaviors of $Y_{t}$ with $c \in[0.8,1.2]$

Figure 4 The dynamical behaviors of $S_{t}-c, I_{t}-c$, and $Y_{t}-c$ with $b=0.2, d=0.2, k=0.2, m=0.5, r=3$, $\beta=0.2, K=10$, and $c \in[0.5,2.5]$, and the initial value $\left(S_{0}, I_{0}, Y_{0}\right)=(6,2,1)$ for model $(1.2)$.

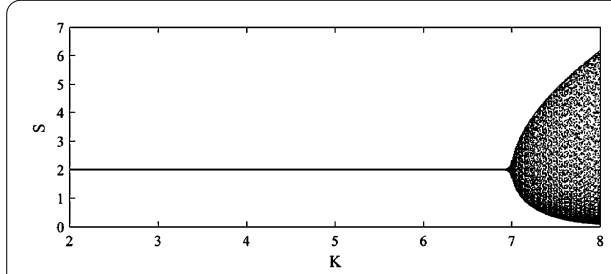

(A) Hopf bifurcation of $S_{t}$ with $K \in[2,8]$

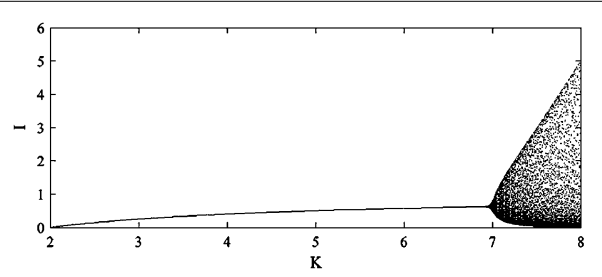

(B) Hopf bifurcation of $I_{t}$ with $K \in[2,8]$

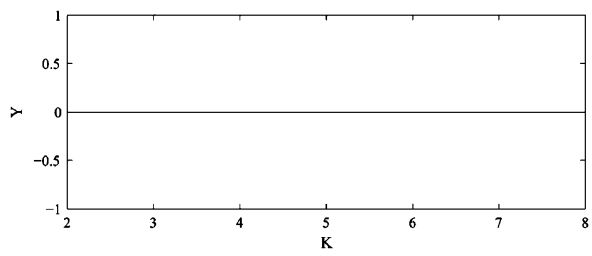

(C) Dynamical behavior of $Y_{t}$ with $K \in[2,8]$

Figure 5 The dynamical behaviors of $S_{t}-K, I_{t}-K$, and $Y_{t}-K$ with $b=0.1, c=0.4, d=0.2, k=0.2$, $m=0.5, r=0.2, \beta=0.2$, and $K \in[2,8]$, and the initial values $\left(S_{0}, I_{0}, Y_{0}\right)=(1,0.5,0.2)$ for model $(1.2)$.

$\left(S_{0}, I_{0}, Y_{0}\right)=(1,0.5,0.2)$. It is obvious that $(b, c, d, k, m, r, \beta, K)=(0.1,0.4,0.2,0.2,0.5,0.2$, $0.2,7) \in P$. Then we have equilibrium $E_{2}\left(\frac{c}{\beta}, \frac{r K}{r+K \beta}\left(1-\frac{c}{K \beta}\right), 0\right)=E_{2}\left(2,1-\frac{3}{1+K}, 0\right)$, and there exists a Hopf bifurcation (Figure 5).

From Figure 5(A) and (B), we see that equilibrium $E_{2}\left(2,1-\frac{3}{1+K}, 0\right)$ is stable when $2 \leq K<$ 7 and loses stability when $K=7$. Further, when $K>7$ there appears a Hopf bifurcation of equilibrium $E_{2}\left(2,1-\frac{3}{1+K}, 0\right)$. However, $Y_{t}$ is in extinction for any values of $K$ ultimately.

Example 5 For detecting the dynamical behaviors of model (1.2) with equilibrium $E_{2}\left(\frac{c}{\beta}, \frac{r K}{r+K \beta}\left(1-\frac{c}{K \beta}\right), 0\right)$ impacted by parameter $\beta$ (the transmission coefficient), we choose $b=0.15, c=0.5, d=0.2, k=0.2, m=0.3, r=4, K=4, \beta \in[0.125,0.42]$, and the initial value $\left(S_{0}, I_{0}, Y_{0}\right)=(2,1,1)$. It is obvious that $(b, c, d, k, m, r, K, \beta)=(0.15,0.5,0.2,0.2,0.3,4$, 


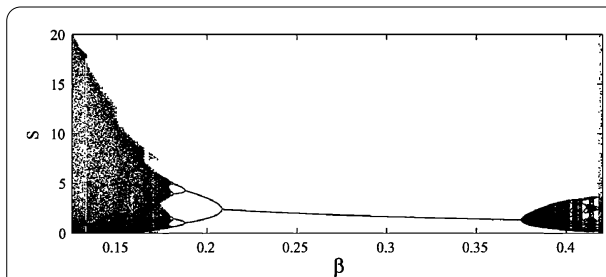

(A) Dynamical behaviors of $S_{t}$ with $\beta \in[0.125,0.42]$

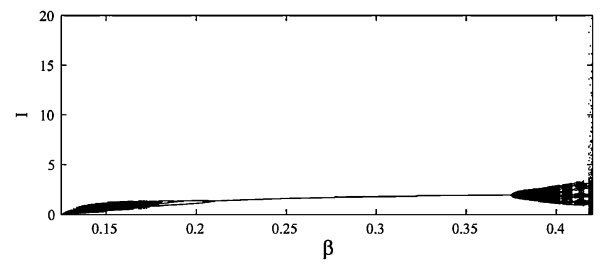

(B) Dynamical behaviors of $I_{t}$ with $\beta \in[0.125,0.42]$

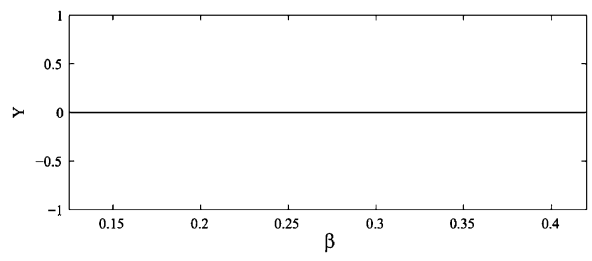

(C) Dynamical behavior of $Y_{t}$ with $\beta \in[0.125,0.42]$

Figure 6 The dynamical behaviors of $S_{t}-\beta, I_{t}-\beta$, and $Y_{t}-\beta$ with $b=0.15, c=0.5, d=0.2, k=0.2$, $m=0.3, r=4, K=4, \beta \in[0.125,0.42]$, and the initial value $\left(S_{0}, I_{0}, Y_{0}\right)=(2,1,1)$ for model (1.2).

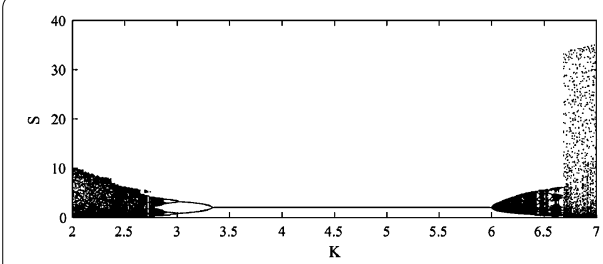

(A) Dynamical behaviors of $S_{t}$ with $K \in[2,7]$

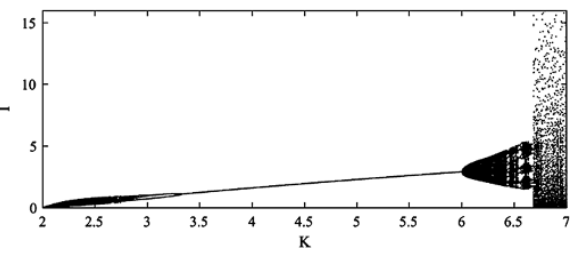

(B) Dynamical behaviors of $I_{t}$ with $K \in[2,7]$

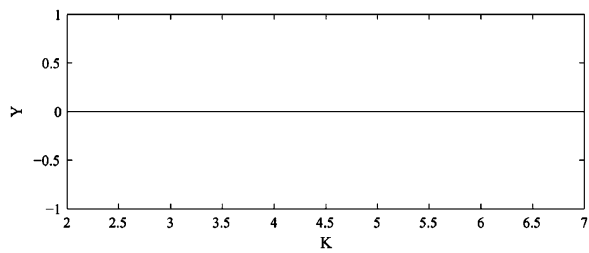

(C) Dynamical behavior of $Y_{t}$ with $K \in[2,7]$

Figure 7 The dynamical behaviors of $S_{t}-K, I_{t}-K$, and $Y_{t}-K$ with $b=0.15, c=0.5, d=0.2, k=0.2$, $m=0.3, r=4, \beta=0.25, K \in[2,7]$, and the initial value $\left(S_{0}, I_{0}, Y_{0}\right)=(1,0.5,0.2)$ for model $(1.2)$.

$4,0.2083),(0.15,0.5,0.2,0.2,0.3,4,4,0.3750) \in N, P$, respectively. Then we have equilibrium $E_{2}\left(\frac{c}{\beta}, \frac{r K}{r+K \beta}\left(1-\frac{c}{K \beta}\right), 0\right)=E_{2}\left(\frac{1}{2 \beta}, \frac{8 \beta-1}{2 \beta}, 0\right)$. From conclusion (2) of Theorem 3, the two bifurcation values are computed as $\beta=0.2083$ and $\beta=0.3750$ from $K \beta=\frac{c r(2+c)}{4+c r}$ and $K \beta=1+c$, respectively. Further, the interval $[0.125,0.42]$ includes the two bifurcation values. Therefore, there appears two bifurcations: flip bifurcation and a Hopf bifurcation. Figure 6(A) and (B) verifies it. In detail, when $\beta$ changes from 0.125 to 0.45 , there appears chaos, flip bifurcation, local stability, Hopf bifurcation, and chaos of $S_{t}$ and $I_{t}$. However, $Y_{t}$ becomes in extinction because of $b k<d$ (Figure 6(C)). On the other hand, the same results appear for $K \in[2,7]$ with $b=0.15, c=0.5, d=0.2, k=0.2, m=0.3, r=4, \beta=0.25$, and the initial value $\left(S_{0}, I_{0}, Y_{0}\right)=(1,0.5,0.2)$ (Figure 7 ). 


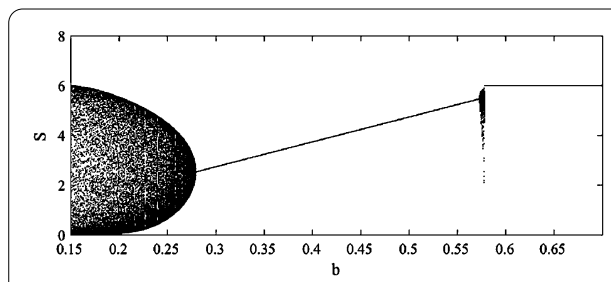

(A) Dynamical behaviors of $S_{t}$ with $b \in[0.15,0.7]$

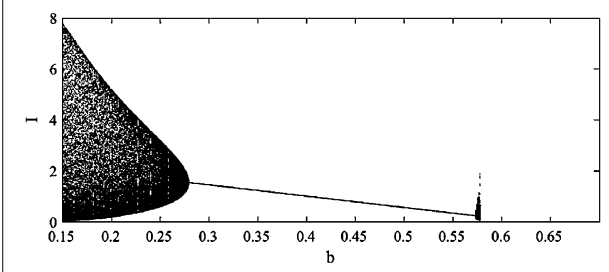

(C) Dynamical behavior of $I_{t}$ with $b \in[0.15,0.7]$

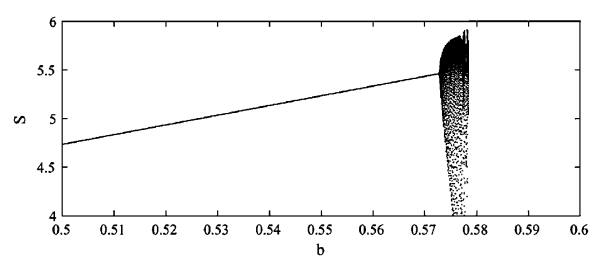

(B) The local amplification of $S_{t}$ with $b \in[0.5,0.6]$

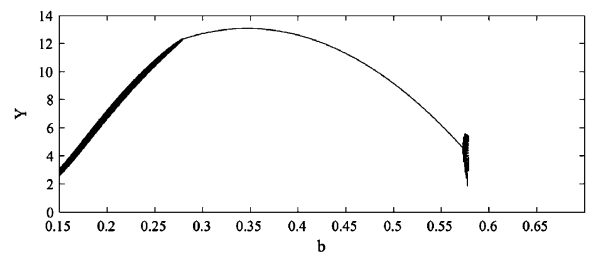

(D) Dynamical behaviors of $Y_{t}$ with $b \in[0.15,0.7]$

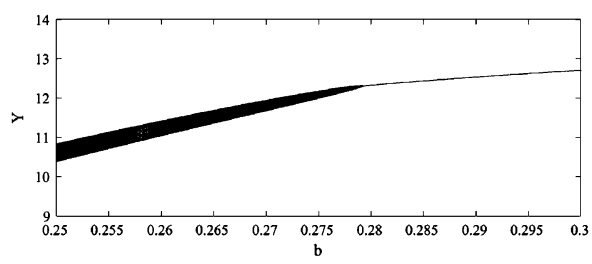

(E) The local amplification of $Y_{t}$ with $b \in[0.25,0.3]$.

Figure 8 The dynamical behaviors of $S_{t}-b, I_{t}-b$, and $Y_{t}-b$ with $c=0.1, d=0.02, k=0.3, m=0.4$, $r=1.2, \beta=0.25, K=6$, and $b \in[0.15,0.7]$, and the initial value $\left(S_{0}, I_{0}, Y_{0}\right)=(2,1.5,1)$ for model $(1.2)$.

Example 6 For detecting the dynamical behaviors of model (1.2) with endemic equilibrium $E^{*}\left(S^{*}, I^{*}, Y^{*}\right)$ impacted by parameter $b$ (the predation coefficient), we choose $c=0.1$, $d=0.02, k=0.3, m=0.4, r=1.2, \beta=0.25, K=6$, and $b \in[0.15,0.7]$, and the initial value $\left(S_{0}, I_{0}, Y_{0}\right)=(2,1.5,1)$. It is obvious that the parameters $c=0.1, d=0.02, k=0.3$, $m=0.4, r=1.2, \beta=0.25, K=6$, and $b=0.28,0.5725$, respectively, satisfy the conditions of conclusion (2) of Theorem 5 . Then we have endemic equilibrium $E^{*}\left(S^{*}, I^{*}, Y^{*}\right)=$ $\left(\frac{150 b-4}{15}, \frac{376-600 b}{135}, \frac{(15 b-1)(188-300 b)}{27}\right)$. Obviously, when $0<b<\frac{188}{300}$ there exists an endemic equilibrium $E^{*}\left(S^{*}, I^{*}, Y^{*}\right)$ of model (1.2).

From Figure 8 we see that when $0.15<b<b_{*}$ there appear chaos and a Hopf bifurcation for model (1.2); when $b_{*} \approx 0.28<b<b_{* *} \approx 0.5725$ the endemic equilibrium $E^{*}\left(S^{*}, I^{*}, Y^{*}\right)$ is stable; when $b_{* *} \approx 0.5725<b<b_{* * *} \approx 0.5785$ there appear Hopf bifurcation and chaos. Furthermore, $S_{t}$ reaches the $K$ value when $b_{* * *} \approx 0.5785<b$, which can be seen from Figure $8(\mathrm{~A})$ and $(\mathrm{B})$. However, $I_{t}$ and $Y_{t}$ become in extinction when $b \approx 0.57$ from Figure $8(\mathrm{C})-(\mathrm{E})$.

Example 7 For detecting the dynamical behaviors of model (1.2) with endemic equilibrium $E^{*}\left(S^{*}, I^{*}, Y^{*}\right)$ impacted by parameter $k$ (the coefficient in conversing prey into predator), we choose $b=0.3, c=0.1, d=0.04, m=0.3, r=1.2, \beta=0.25, K=6$, and $k \in[0.15,1]$, and the initial value $\left(S_{0}, I_{0}, Y_{0}\right)=(2,1.5,1)$. It is obvious that parameters $b=0.3, c=0.1, d=0.04, m=0.3, r=1.2, \beta=0.25, K=6$, and $k=0.34$ satisfy the conditions of conclusion (4) of Theorem 5. Then we have endemic equilibrium $E^{*}\left(S^{*}, I^{*}, Y^{*}\right)=$ 


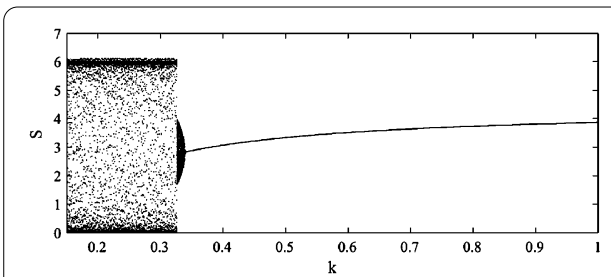

(A) Dynamical behaviors of $S_{t}$ with $k \in[0.15,1]$

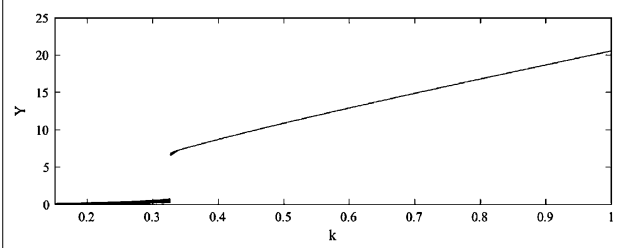

(C) Dynamical behaviors of $Y_{t}$ with $k \in[0.15,1]$

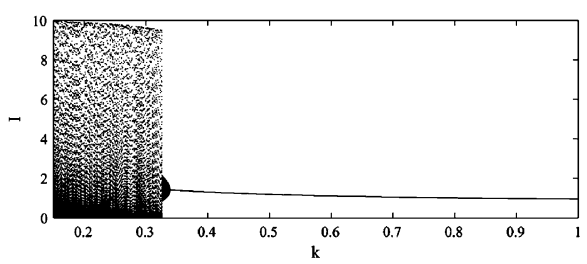

(B) Dynamical behaviors of $I_{t}$ with $k \in[0.15,1]$

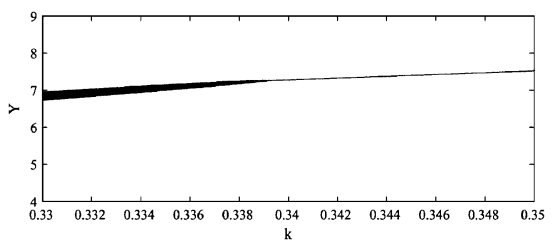

(D) The local amplification of $Y_{t}$ with $k \in[0.33,0.35]$

Figure 9 The dynamical behaviors of $S_{t}-k, I_{t}-k$, and $Y_{t}-k$ with $b=0.3, c=0.1, d=0.04, m=0.3$, $r=1.2, \beta=0.25, K=6$, and $k \in[0.15,1]$, and the initial value $\left(S_{0}, I_{0}, Y_{0}\right)=(2,1.5,1)$ for model $(1.2)$.

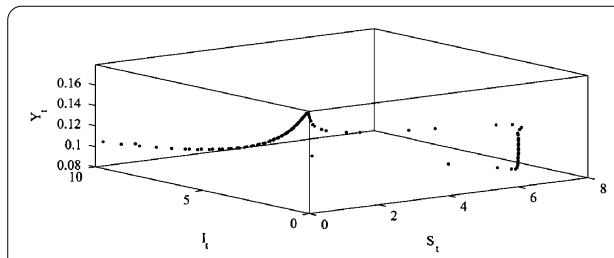

(A) $k=0.2$

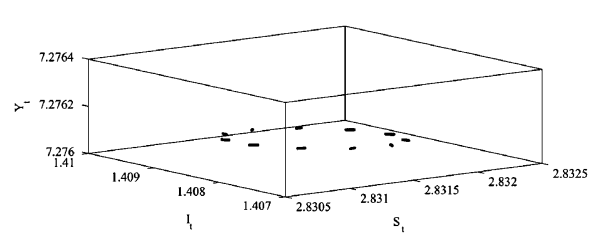

(B) $k=0.34$

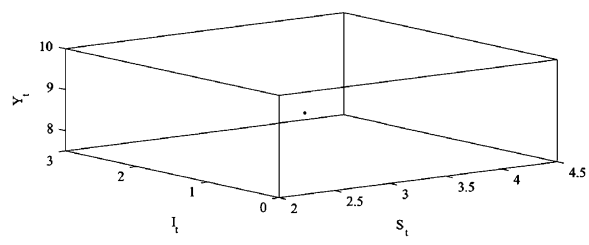

(C) $k=0.4$

Figure 10 The phase portraits of model (1.2) with $k=0.2,0.34,0.4$ corresponding to Figure 9 .

$\left(\frac{66 k-8}{15}, \frac{392-264 k}{135}, \frac{(15 k-2)(392-264 k)}{81}\right)$. Obviously, when $0.15 \leq k \leq 1$ there exists an endemic equilibrium $E^{*}\left(S^{*}, I^{*}, Y^{*}\right)$.

Figure 9 shows that when $0.15<k<k_{*} \approx 0.34$ there exist chaos and a Hopf bifurcation for $S_{t}, I_{t}$, and $Y_{t}$. With the increasing of $k$ from $k_{*} \approx 0.34$ to 1 , the numbers of $S_{t}$ and $Y_{t}$ are increasing while $I_{t}$ is decreasing. And this result agrees with the ecological significance.

For better understanding of the above results, we only provide phase portraits (see Figure 10) of model (1.2) under the condition $k=0.2,0.34,0.4$ in Example 7. The other cases are similar to Example 7 and we omit them here.

Remark 3 From Subcase 2 of Example 3, when we choose the same parameters and the initial value, then we vary the parameter $c$ we see that there appears a series complex 
dynamical behavior in equilibrium $E_{2}\left(\frac{c}{\beta}, \frac{r K}{r+K \beta}\left(1-\frac{c}{K \beta}\right), 0\right)$ of model (1.2), such as chaos $\rightarrow$ flip bifurcation $\rightarrow$ local stability $\rightarrow$ Hopf bifurcation $\rightarrow$ chaos too.

Remark 4 From Example 6, when we choose the same parameters and the initial value, then when we vary the parameter $b$ we see that there appears a series complex dynamical behaviors in equilibrium $E^{*}\left(S^{*}, I^{*}, Y^{*}\right)$ of model (1.2), such as chaos $\rightarrow$ Hopf bifurcation $\rightarrow$ local stability $\rightarrow$ Hopf bifurcation $\rightarrow$ chaos too.

Remark 5 The predation coefficient $b$ of predator plays an important role in biological disease prevention and cure which can be seen in Example 6. And this is significant in the actual situation of ecology.

Open problem 1 Whether there exist some special population models when we fix same parameters and vary one special parameter there appears a series bifurcations and chaos.

Open problem 2 From the above discussion, whether there exists chaos $\rightarrow$ flip bifurcation $\rightarrow$ local stability $\rightarrow$ flip bifurcation $\rightarrow$ chaos in some special population model when some parameters are fixed at the same values and one parameter is varied continuously. This will be our future study.

\section{Discussion}

The study about discrete-time eco-epidemiological model is payed little attention in previous works. In this paper, we discussed the dynamical behaviors of a discrete-time ecoepidemiological model (1.2). The threshold parameter $R_{0}$ is obtained which controls the development of the disease. When $R_{0}<1,0<r \leq 1$ (or $R_{0} \frac{\exp [r-1]}{r}<1,1<r<2$ ) the susceptible prey reaches the carrying capacity, while the infected prey and the predator become in extinction. It implies that the disease dies out in the prey population. When $R_{0}>1$ and $k b<d$ the susceptible prey and infected prey coexist, while the predator becomes in extinction. This implies that the disease persists in the prey and the paradoxical phenomenon is obtained that sufficient enrichment of resources leads to extinction of the predator.

Further, the different complex dynamical behaviors of model (1.2) caused by different parameters have been investigated using the difference equation theories in ecological perspective: such as flip bifurcation, Hopf bifurcation, chaos, and more complex dynamical behaviors. This is far richer than the corresponding continuous-time model (1.1). The results now are given in detail.

1. For the parameters $b$ (the predation coefficient), $k$ (the coefficient in conversing prey into predator) and $d$ (the death rate constant of the predator), when they satisfy $b k<d$ the predator $Y_{t}$ always becomes in extinction, which agrees with the ecological phenomenon.

2. For equilibrium $E_{2}\left(\frac{c}{\beta}, \frac{r K}{r+K \beta}\left(1-\frac{c}{K \beta}\right), 0\right)$ of model (1.2), we choose four key parameters $r, K, \beta, c$ that directly influence the dynamical behaviors of model (1.2). When they vary at different values model (1.2) appears local stability, flip bifurcation, Hopf bifurcation, and chaos. Further, the most interesting aspect is choosing the same parameters and the initial value of model (1.2); then $K$ or $\beta$ shows a continuous increase from some fixed value above 0 to $\frac{c r(2+c)}{4+c r}$ then to $1+c$, then there appears a series of dynamical behaviors: chaos $\rightarrow$ flip bifurcation $\rightarrow$ local stability $\rightarrow$ Hopf bifurcation $\rightarrow$ chaos too which is the same result as in [2]. Hence, there is an interesting open problem when we fix the same parameters and 
vary one special parameter: whether there exist series bifurcations and chaos for other population models.

Moreover, when we choose the same parameters and the initial value of model (1.2); then $c$ has a continuous increase from some fixed value above 0 to $K \beta-1$ then to $c_{2}$, then there appears a series of dynamical behaviors: chaos $\rightarrow$ Hopf bifurcation $\rightarrow$ local stability $\rightarrow$ flip bifurcation $\rightarrow$ chaos too (Figure 4). This phenomenon is rarely seen in previous work.

3. For positive equilibrium $E^{*}\left(S^{*}, I^{*}, Y^{*}\right)$ of model (1.2), we choose the key parameters $b$ (the predation coefficient) and $k$ (the coefficient in conversing prey into predator) to detect the variation of the dynamical behaviors. When the parameter $b$ changes at different values and other parameters are fixed at the same values there appears a series of dynamical behaviors: chaos $\rightarrow$ Hopf bifurcation $\rightarrow$ local stability $\rightarrow$ Hopf bifurcation $\rightarrow$ chaos too, which can be seen from Figure 8. When parameter $k$ increases the number of $I_{t}$ is decreasing while $S_{t}$ and $Y_{t}$ are increasing and there appears Hopf bifurcation of model (1.2) when $k=k^{*}$ (Figure 9).

Generally, the predation ability and conversing prey into predator ability are important to influence the numbers of predator and infected prey. In this study, the results imply that when the parameter $b$ or $k$ is increasing, then the number of predator $Y_{t}$ is increasing, while $I_{t}$ is decreasing. And this will be of significance in controlling the disease of prey in an eco-epidemiological model.

However, there are still many interesting and challenging questions which need to be studied for model (1.2). For example, we only obtain the local stability, bifurcations, and chaos dynamical behaviors of model (1.2). We can ask the questions: Whether the global asymptotic stability of model (1.2) can be obtained. And if model (1.2) occurs with different functional responses or different types of incidence rate then how to study the dynamical behaviors. Furthermore, if the susceptible prey is preyed in model (1.2), whether there exist complex dynamical behaviors. We will investigate them in our future work.

Competing interests

The authors declare that they have no competing interests.

Authors' contributions

The authors declare that the study was realized in collaboration with the same responsibility. All authors read and approved the final manuscript.

\section{Author details}

'State Key Laboratory of Desert and Oasis Ecology, Xinjiang Institute of Ecology and Geography, Chinese Academy of Sciences, Urumqi, 830011, People's Republic of China. ${ }^{2}$ College of Mathematics and System Sciences, Xinjiang University, Urumqi, 830046, People's Republic of China.

\section{Acknowledgements}

This work was supported by the National Natural Science Foundation of P.R. China (11401569, 11271312), International Science \& Technology Cooperation Program of China (2010DFA92720) and the Natural Science Foundation of Xinjiang (2014211B047).

Received: 7 June 2014 Accepted: 24 September 2014 Published: 14 Oct 2014

\section{References}

1. Hassell, MP, Comins, HN, Mayt, RM: Spatial structure and chaos in insect population dynamics. Nature 353, 255-258 (1991)

2. Allesina, S, Tang, S: Stability criteria for complex ecosystems. Nature 483, 205-208 (2012)

3. Bergerud, AT: Prey switching in a simple ecosystem. Sci. Am. 249, 130-141 (1983)

4. Mori, T, Saitoh, T: Flood disturbance and predator-prey effects on regional gradients in species diversity. Ecology 95 132-141 (2014)

5. Trainor, AM, Schmitz, OJ, Ivan, JS, Shenk, TM: Enhancing species distribution modeling by characterizing predator-prey interactions. Ecol. Appl. 24, 204-216 (2014) 
6. Kao, RR, Gravenor, MB, Baylis, M Bostock, CJ, Chihota, CM, Evans, JC, Goldmann, W, Smith, AJ, McLean, AR: The potential size and duration of an epidemic of bovine spongiform encephalopathy in British sheep. Science 295 332-335 (2002)

7. Van der Plank, JE: Dynamics of epidemics of plant disease. Science 147, 120-124 (1965)

8. Sanson, RL, Dube, C, Cork, SC, Frederickson, R, Morley, C: Simulation modelling of a hypothetical introduction of foot-and-mouth disease into Alberta. Prev. Vet. Med. 114, 151-163 (2014)

9. Ashrafur Rahmana, SM, Vaidy, N, Zou, X: Impact of Tenofovir gel as a PrEP on HIV infection: a mathematical model. J. Theor. Biol. 347, 151-159 (2014)

10. Souza, MO: Multiscale analysis for a vector-borne epidemic model. J. Math. Biol. 68, 1269-1293 (2014)

11. Kaiser, J: Salton sea: battle over a dying sea. Science 284, 28-30 (1999)

12. Slack, G: Salton sea sickness. Pac. Discovery 50, 3-5 (1997)

13. Anderson, RM, May, R: The invasion, persistence and spread of infectious diseases within animal and plant communities. Philos. Trans. R. Soc. Lond. B, Biol. Sci. 314, 533-570 (1982)

14. Bairagi, N, Chaudhuri, S, Chattopadhyay, J: Harvesting as a disease control measures in an eco-epidemiological system - a theoretical study. Math. Biosci. 217, 134-144 (2009)

15. Jana, S, Kar, T: A mathematical study of a prey-predator model in relevance to pest control. Nonlinear Dyn. 74 , 667-683 (2013)

16. Sharp, A, Pastor, J: Stable limit cycles and the paradox of enrichment in a model of chronic wasting disease. Ecol. Appl. 21, 1024-1030 (2011)

17. Olsen, LF, Truty, GL, Schaffer, WM: Oscillations and chaos in epidemics: a non-linear dynamic study of six childhood diseases in Copenhagen, Denmark. Theor. Popul. Biol. 33, 344-370 (1988)

18. Chattopadhyay, J, Arino, O: A predator-prey model with disease in the prey. Nonlinear Anal. 36, 747-766 (1999)

19. Hethcote, HW, Wang, W, Han, L, Ma, Z: A predator-prey model with infected prey. Theor. Popul. Biol. 66, 259-268 (2004)

20. Das, KP, Chatterjee, S, Chattopadhyay, J: Disease in prey population and body size of intermediate predator reduce the prevalence of chaos-conclusion drawn from Hastings-Powell model. Ecol. Complex. 6, 363-374 (2009)

21. Greenhalgh, D, Haque, M: A predator-prey model with disease in the prey species only. Math. Methods Appl. Sci. 30 911-929 (2006)

22. Wang, J, Qu, X: Qualitative analysis for a ratio-dependent predator-prey model with disease and diffusion. Appl. Math Comput. 217, 9933-9947 (2011)

23. Xiao, Y, Chen, L: Modeling and analysis of a predator-prey model with disease in the prey. Math. Biosci. 171, 59-82 (2001)

24. Xiao, Y, Chen, L: A ratio-dependent predator-prey model with disease in the prey. Appl. Math. Comput. 131, 397-414 (2002)

25. Li, J, Gao, W: Analysis of a prey-predator model with disease in prey. Appl. Math. Comput. 217, 4024-4035 (2010)

26. Venturino, E: Epidemics in predator-prey model: disease in the predators. IMA J. Math. Appl. Med. Biol. 19, 185-205 (2002)

27. Bob, WK, George, AK, Krishna, PD: Stabilization and complex dynamics in a predator-prey model with predator suffering from an infectious disease. Ecol. Complex. 8, 113-122 (2011)

28. Auger, P, Mchich, R, Chowdhury, T, Sallet, G, Tchuente, M, Chattopadhyay, J: Effects of a disease affecting a predator on the dynamics of a predator-prey system. J. Theor. Biol. 258, 344-351 (2009)

29. Wilmers, C, Post, E, Peterson, R, Vucetich, J: Predator-disease out-break modulates top-down, bottom-up and climatic effects on herbivore population dynamics. Ecol. Lett. 9, 383-389 (2006)

30. Haque, M: A predator-prey model with disease in the predator species only. Nonlinear Anal., Real World Appl. 11 2224-2236 (2010)

31. Das, K, Kundu, K, Chattopadhyay, J: A predator-prey mathematical model with both the populations affected by diseases. Ecol. Complex. 8, 68-80 (2011)

32. Gao, S, Chen, L: The effect of seasonal harvesting on a single-species discrete population model with stage structure and birth pulse. Chaos Solitons Fractals 24, 1013-1023 (2005)

33. Hu, Z, Teng, Z, Zhang, L: Stability and bifurcation analysis of a discrete predator-prey model with nonmonotonic functional response. Nonlinear Anal., Real World Appl. 12, 2356-2377 (2011)

34. Chen, G, Teng, Z, Hu, Z: Analysis of stability for a discrete ratio-dependent predator-prey system. Indian J. Pure Appl. Math. 42,1-26 (2011)

35. Teng, Z, Zhang, Y, Gao, S: Permanence criteria for general delayed discrete nonautonomous $n$-species Kolmogorov systems and its applications. Comput. Math. Appl. 59, 812-828 (2010)

36. Liu, X, Xiao, D: Complex dynamical behaviors of a discrete-time predator-prey system. Chaos Solitons Fractals 32 , 80-94 (2007)

37. Agiza, HN, Elabbasy, EM, El-Metwally, H, Elsadany, AA: Chaotic dynamical of a discrete prey-predator model with Holling type II. Nonlinear Anal. 10, 116-129 (2009)

38. Celik, C, Duman, O: Allee effect in a discrete-time predator-prey system. Chaos Solitons Fractals 40, 1956-1962 (2009)

39. Basson, M, Fogarty, MJ: Harvesting in discrete-time predator-prey systems. Math. Biosci. 141, 41-74 (1997)

40. Chen, $X$ : Periodicity in a nonlinear discrete predator-prey system with state dependent delays. Nonlinear Anal., Rea World Appl. 8, 435-446 (2007)

41. Fan, Y, Li, W: Permanence for a delayed discrete ratio-dependent predator-prey system with Holling type functional response. J. Math. Anal. Appl. 299, 357-374 (2004)

42. Fan, SJ: A new extracting formula and a new distinguishing means on the one variable cubic equation. J. Hainan Teach. Coll. 2, 91-98 (1989) (in Chinese)

43. Hu, Z, Teng, Z, Jiang, H: Stability analysis in a class of discrete SIRS epidemic models. Nonlinear Anal., Real World Appl. 13, 2017-2033 (2012)

10.1186/1687-1847-2014-265

Cite this article as: Hu et al.: Complex dynamical behaviors in a discrete eco-epidemiological model with disease in

prey. Advances in Difference Equations 2014, 2014:265 